\title{
ASSOCIATION OF VARIANTS IN THE $C P$, ATOXI AND COMMD1 GENES WITH WILSON DISEASE SYMPTOMS IN LATVIA
}

\author{
Zarina $\mathrm{A}^{1,2^{*}}$, Tolmane $\mathrm{I}^{3,4}$, Krumina $\mathrm{Z}^{2}$, Tutane $\mathrm{AI}^{1}$, Gailite $\mathrm{L}^{1}$ \\ *Corresponding Author: Ms. Agnese Zarina, Scientific Laboratory of Molecular Genetics, Rīga Stradiņš \\ University, 16 Dzirciema Street, Riga, Latvia, LV-1007. Tel.: +371-6706-1542. Fax: +371-67-471-815. \\ E-mail: agnese.zarina@rsu.lv or zarina.agnese@gmail.com
}

\begin{abstract}
Wilson's disease (WD) is a copper metabolism disorder, caused by allelic variants in the $A T P 7 B$ gene. Wilson's disease can be diagnosed by clinical symptoms, increased copper and decreased cerulopasmin levels, which could all also be by other genetic variants beyond the $A T P 7 B$ gene, e.g., disturbed ceruloplasmin biosynthesis can be caused by pathogenic allelic variants of the $C P$ gene. Copper metabolism in the organism is affected by several molecules, but pathogenic variants and related phenotypes are described with $C O M M D 1$ and $A T O X 1$ genes. The aim of the study was to test other genes, $C P, A T O X 1$ and COMMD1, for possible influence to the manifestation of WD. Patients were enrolled on the basis of Leipzig's diagnostic criteria, 64 unrelated patients with confirmed WD. Direct sequencing of promoter region of the $C P$ gene and $A T O X 1$ and $C O M M D 1$ gene exons was conducted. Statistically significant differences were found between the two variants in the $C P$ gene and the $A T P 7 B$ genotype (rs66508328 variant AA genotype and the rs 11708215 variant GG genotype) were more common in WD patients with an unconfirmed $A T P 7 B$ genotype. One allelic (intronic) variant was found in the $A T O X 1$ gene without causing the functional changes of the gene. Three allelic variants were identified in the COMMD1 gene. No statistically significant differences were found between allele and genotype frequencies and the first clinical manifestations of WD. Different variants of the $C P$ gene contributed to a WD-like phenotype in clinically confirmed WD patients with neurological
\end{abstract}

\footnotetext{
${ }^{1}$ Scientific Laboratory of Molecular Genetics, Rīga Stradiņš University, Riga, Latvia

2 Department of Biology and Microbiology, Rīga Stradiņš University, Riga, Latvia

${ }^{3}$ Riga East Clinical University Hospital, stationary "Latvian Centre of Infectious Diseases", Riga, Latvia

${ }^{4}$ Faculty of Medicine, University of Latvia, Riga, Latvia
}

symptoms and without identified pathogenic variants in the $A T P 7 B$ gene. Allelic variants in the ATOX1 and COMMDI genes do not modify the clinical manifestation of WD in Latvian patients. (266 words)

Keywords: Copper metabolism; Decreased blood ceruloplasmin; Wilson's disease (WD).

\section{INTRODUCTION}

Wilson's disease (WD) is an autosomal recessive disorder of copper metabolism, caused by allelic variants of the $A T P 7 B$ gene. Wilson's disease has been established as a monogenic disorder, although heterogeneity in phenotype is observed even in patients possessing the same type of allelic variants in the $A T P 7 B$ gene, leading to the assumption about other genetic modifiers affecting the WD phenotype [1]. There are many studies looking for factors modifying the clinical presentation of WD, such as allelic variants in the genes COMMD1, ATOX1, XIAP, APOE DMT1 (SLC11A2), ATP7A, MTHFR, ESD, INO80 and $P R N P$ genes, as well as changes in epigenetic mechanisms of gene expression regulation [2-4]. Moreover, as the main pathogenetic mechanism is copper metabolism disorder, in this study we analyzed pathogenic variants in the genes affecting copper metabolism; there are previously described pathogenic variants and related phenotypes with the COMMD1 and ATOX1 genes [5,6].

One of the criteria of WD diagnostics is decreased ceruloplasmin level. Disturbed ceruloplasmin biosynthesis caused by pathogenic allelic variants of the $C P$ gene leads to decreased ceruloplasmin level in the blood, which disrupts iron metabolism, resulting in iron accumulation in various organs, especially basal ganglia, causing serious neuronal damage. Iron accumulation in the brain leads to neurodegeneration and neurological symptoms such as motor disorientation and other motor deficits in the age range of 45 to 55 years [7]. The association of the $C P$ gene allelic 
variants and different diseases is described in various studies of Parkinson's disease [8] and atrial fibrillation (AFib) $[9,10]$, concluding that changes in the $C P$ gene promoter may be associated with altered levels of ceruloplasmin.

\section{MATERIALS AND METHODS}

This study protocol was approved by the Latvian Central Medical Ethics Comittee and was performed according to the Declaration of Helsinki. Informed consent was obtained from all patients. In the study, 64 unrelated patients with confirmed WD, were enrolled on the basis of Leipzig's diagnostic criteria [11]. All patients included in the study carried at least four points according to the WD scoring system. According to the initial symptoms, WD patients were categorized in the following groups, as described elsewhere [12]: asymptomatic, hepatic, neurological/psychiatric and neurological/hepatic.

Genomic DNA was extracted from peripheral blood by a standard phenol/chloroform method. Allelic variant c.3207 C>A; p.H1069Q (rs76151636) was tested by polymerase chain reaction (PCR)-BiPASA (bidirectional PCR of specific alleles) [13].

Direct DNA sequencing of the ATP7B gene (promoter, exons and exon-intron boundaries) was performed for the patients with WD symptoms, being either heterozygous for H1069Q or without it on any allele. Methods for WD molecular confirmation and the group of WD patients have been described before in detail $[14,15]$.

Direct Sequences of the $A T O X 1, C O M M D 1$ and $C P$ Genes. For sequencing of the $C P$ gene promoter region, ATOX1 and COMMD1 gene exons and adjacent introns, primers selected using the open access Primer 3 program (available at http://bioninfo.ut.ee/primer3-0.4.0/) and previously reported primers were used $[6,8]$. For the genetic variants nomenclature, traditional names were used (if existing), or HGVS nomenclature according to reference sequences: $C P$ gene (NM_000096.3, NP_000087.1); ATOX1 gene (NM_004045.3, NP_004036.1); COMMD1 gene (NM 152516.3, NP 689729.1). Allele frequencies in the European population were obtained from GnomAD database (https:/gnomad.broadinstitute.org/) [16]. For Link- age data, the 1000 Genome browser (https://www. international genome.org) was used [17]. If a variant was not reported to have a functional effect, it was characterized according to the American College of Medical Genetics and Genomics (ACMG guidelines [18], including in silico tools. The $C P$ gene promoter region (not all of the gene) sequencing was selected based on information in other studies concluding that changes in the $C P$ gene promoter may be associated with altered levels of ceruloplasmin [8].

Statistical Analyses. Data processing was performed using the Statistical Package for the Social Sciences (IBM SPSS $($ ) version 22 (https://www.ibm.com/SPSS-Statistics/Software), and PLINK 1.07 (http://zzz.bwh.harvard. edu/plink/). The comparison between patient groups was done by Fisher exact $t$-test. The frequencies of genotypes in the WD patient population before further data analysis were tested for Hardy-Weinberg equilibrium. The association of the COMMDI and $C P$ gene variants with WD phenotype was analyzed in the allelic, dominant, recessive and genotypic inheritance model.

\section{RESULTS}

The majority of the WD patients expressed hepatological symptoms as the first manifestation (detailed segregation of the WD patients according to their first symptoms and age of onset can be found in Table 1). A detailed description of WD patient genotypes in the $A T P 7 B$ gene, clinical and biochemical findings can be found in the Supplemental Table 1. All identified variants corresponded to the Hardy-Weinberg equilibrium in the analyzed WD patient group $(p>0.05)$.

The $\boldsymbol{C P}$ Gene. In the $C P$ gene promoter, seven allelic variants were observed: rs66508328, rs67870152, rs16861642, rs73020328, rs73166855, rs66953613 and rs 11708215 . As the first six of the variants mentioned above are in strong linkage $\left(r^{2}=1\right)$ (see Table 2), one of them was analyzed, rs66508328 along with rs11708215 $\left(r^{2}\right.$ $=0.562$ from the 1000 genome project) [17], the frequency of which differed from the other variants.

None of the variants in the WD patients was statistically significantly different from those described in the GnomAD

Table 1. Segregation of Wilson's disease patients in the Latvian population according to their clinical findings.

\begin{tabular}{|l|l|l|l|c|}
\hline Parameters & $\begin{array}{c}\text { Hepatologiacal } \\
(\boldsymbol{n}=\mathbf{4 2})\end{array}$ & $\begin{array}{l}\text { Neurological and/or } \\
\text { Psychiatric }(\boldsymbol{n}=\mathbf{1 7})\end{array}$ & $\begin{array}{l}\text { Mixed } \\
(\boldsymbol{n}=\mathbf{5})\end{array}$ & $\boldsymbol{p}$ Value \\
\hline $\begin{array}{l}\text { Level of ceruloplasmin in blood (g/dL) } \\
\text { [median (IQR)] }\end{array}$ & $\begin{array}{l}0.11 \\
(0.08-0.13)\end{array}$ & $\begin{array}{l}0.13 \\
(0.08-0.15)\end{array}$ & $\begin{array}{l}0.08 \\
(0.07-0.15)\end{array}$ & 0.562 \\
\hline 24-hour urine copper [median (IQR)] & $\begin{array}{l}197.0 \\
(136.0-373.0)\end{array}$ & $\begin{array}{l}163.0 \\
(96.0-268.0)\end{array}$ & $\begin{array}{l}374.0 .0 \\
(365.0-384.0)\end{array}$ & 0.370 \\
\hline Age of onset [average ( $\pm \mathrm{SD})]$ & $20.21( \pm 9.10)$ & $29.82( \pm 13.25)$ & $36.00( \pm 8.75)$ & 0.066 \\
\hline
\end{tabular}

IQR: interquartile range; SD: standard deviation. 
database. Next, the frequency of alleles and genotypes (according to different inheritance models) was compared with the first clinical manifestation and genotype of the $A T P 7 B$ gene (see Table 3). No statistically significant differences were found between allele frequencies and the first clinical manifestations of WD. Statistically significant differences were found between the two analyzed variants and the ATP7B genotype, rs66508328 variant AA genotype and the rs 11708215 variant $G G$ genotype (both according to recessive inheritance model) were more common in WD patients with the unconfirmed genotype by $A T P 7 B$ gene molecular testing.

The ATOX1 Gene. Four patients (out of 64) were found to have one allelic (intronic) variant, rs571657964 in a heterozygous state [minor alle frequency (MAF) for patients $=0.023$; MAF in European (non Finnish) population $=0.0058]$ [16]. Analyzing the variant in the Human Splicing Finder software (http://www.umd.be/HSF/) [19], it was predicted that the allelic variant had no effect on splicing, thus the allelic variant was not included in further statistical analyses, as it was very unlikely to cause functional changes of the $A T O X 1$ gene.

The COMMD1 Gene. Three allelic variants were identified: rs569267407, rs55677935 and rs9096. The frequency of allelic variants of the COMMD1 gene found in this study was compared to the GnomAD database. See results in Table 4 .

Table 2. Frequency of two selected allelic variants of the $C P$ gene promoter identified in Wilson's disease patients.

\begin{tabular}{|l|c|c|}
\hline Parameters & $\mathbf{r s 6 6 5 0 8 3 2 8}$ & rs11708215 \\
\hline Alleles & $\mathrm{G}>\mathrm{A}$ & $\mathrm{A}>\mathrm{G}$ \\
\hline MAF (this study) & 0.0902 & 0.1750 \\
\hline MAF (GnomAD), European (non Finnish population) & 0.1068 & 0.2078 \\
\hline$p$ Value (odds ratio) & $0.550(1.2070)$ & $0.3771(1.2369)$ \\
\hline Genotypes & $2: \mathrm{AA} ; 7: \mathrm{AG} ; 52: \mathrm{GG}$ & $2: \mathrm{GG} ; 17: \mathrm{GA} ; 41: \mathrm{AA}$ \\
\hline$p$ Value & 0.0608 & 1.0000 \\
\hline
\end{tabular}

MAF: minor allele frequency.

Table 3. Relation of the $C P$ gene promoter allelic variants to Wilson's disease phenotype and the $A T P 7 B$ genotype.

\begin{tabular}{|l|l|l|l|c|}
\hline & \multicolumn{1}{|c|}{ Inheritance Model } & \multicolumn{2}{c|}{ ATP7B Gene Genotype } & $p$ Value \\
\hline & & Non $\mathrm{WD}^{\mathrm{a}}(n=12)$ & $\mathrm{WD}^{\mathrm{b}}(n=49)$ & \\
\hline rs66508328 & Genotype (AA/AG/GG) & 2: AA; 0: AG; 10: GG & 0: AA; 7: AG; 42: GG & $\mathbf{0 . 0 2 8 9}$ \\
\hline & Allele (A/G) & $4: \mathrm{A} ; 20: \mathrm{G}$ & 7: A; 91: G & 0.2240 \\
\hline & Dominant (AA+AG/GG) & 2: AA+AG; 10: AG/GG & 7: AA+AG; 42: AG/GG & 1.0000 \\
\hline & Recessive (AA/AG+GG) & 2: AA/AG; 10: AG+GG & 0: AA/AG; 49:AG+GG & $\mathbf{0 . 0 3 6 1}$ \\
\hline rs11708215 & Genotype (GG/GA/AA) & 2: GG; 0: GA; 10: AA & 0: GG; 17: GA; 31: AA & $\mathbf{0 . 0 0 1 6}$ \\
\hline & Allele (G/A) & 4: G; 20: A & 17: G; 79: A & 1.0000 \\
\hline & Dominant (GG+GA/AA) & 2: GG+GA; 10: GA/AA & 17: GG+GA; 31: GA/AA & 0.3059 \\
\hline & Recessive (GG/GA+AA) & 2: GG/GA; 10: GA+AA & 0: GG/GA; 48: GA+AA & $\mathbf{0 . 0 3 7 3}$ \\
\hline
\end{tabular}

${ }^{a}$ Non WD: patients who did not have two pathogenic variants in the $A T P 7 B$ gene.

${ }^{\mathrm{b}} \mathrm{WD}$ : patients with two pathogenic variants in the $A T P 7 B$ gene.

Table 4. Variants found in the COMMD1 gene in Wilson's disease patients.

\begin{tabular}{|l|c|c|c|}
\hline Variant & rs569267407 & rs55677935 & rs9096 \\
\hline Reference: NM_152516.3 & c.-68_67delTT & c.358C>T & c.492C>T \\
\hline Reference: NP_689729.1 & - & p.Arg120Trp & p.Asp164Trp \\
\hline Location in the COMMD1 gene & intron 1 & exon 2 & exon 3 \\
\hline MAF (this study) & 0.0088 & 0.0440 & 0.0968 \\
\hline MAF (GnomAD), European (non Finnish population) & 0.0020 & 0.0215 & 0.1263 \\
\hline$p$ Value & 0.1469 & 0.1553 & 0.3247 \\
\hline
\end{tabular}

MAF: minor allele frequency. 
The frequencies of COMMD1 variants did not significantly differ from the frequencies listed in the GnomAD database. As the variant rs569267407 is located in the non coding part of the gene, its potential effect on gene splicing was analyzed (using the Human Splicing Finder software) [19]. As the impact was not predicted, the variant was not further analyzed. The frequency of alleles or genotypes (by different inheritance models) was compared to the first clinical symptoms of WD patients (taking into account only those patients with molecularly confirmed $\mathrm{WD}, n=49$ ). No statistically significant differences were found between allele and genotype frequencies and the first clinical manifestations of WD. Comparing the incidence of both variants (in the dominant inheritance model) and the age of first symptoms, no differences were observed (rs9096 $p=0.112$; rs55677935 $p=0.146$ ); results are shown in Table 5. diseases, e.g., aceruloplasminemia caused by pathogenic allelic variants in the $C P$ gene. Neurological symptoms of aceruloplasminemia can mimic WD, but this is actually due to the accumulation of iron [20]. In such cases, to confirm aceruloplasminemia, serum ceruloplasmin levels of first-degree relatives should be checked (serum ceruloplasmin is also reduced in heterozygous patients with pathogenic allelic variants), as well as patients with aceruloplasminemia generally found to have reduced serum iron levels, increased ferritin levels, diabetes and evidence of iron accumulation in magnetic resonance imaging in the brain [22]. In view of lack of the aforementioned changes in symptoms and laboratory parameters in WD patients without a molecularly confirmed diagnosis, the diagnosis of aceruloplasminemia is questionable. The association of allelic variants in the $C P$ gene and different diseases are described in various studies: with Parkinson's disease

Table 5. Relation of the allelic variants in the COMMD1 gene to the Wilson's disease phenotype.

\begin{tabular}{|l|c|l|l|l|l|c|}
\hline Variant & Allele 1 & Allele 2 & \multicolumn{1}{|c|}{ Inheritance Model } & \multicolumn{1}{|c|}{$\begin{array}{c}\text { Neurological } \\
\text { Symptoms }(\boldsymbol{n}=7)\end{array}$} & $\begin{array}{c}\text { Hepatological } \\
\text { Symptoms }(\boldsymbol{n}=\mathbf{4 2})\end{array}$ & $\boldsymbol{p}$ Value \\
\hline rs55677935 & $\mathrm{C}$ & $\mathrm{T}$ & Genotype (TT/TC/CC) & 0: TT; 0: TC; 7: CC & 1: TT; 2: TC; 39: CC & 1.0000 \\
\hline & & & Allele (T/C) & 0: T; 14: C & 4: T; 80: C & 1.0000 \\
\hline & & & Dominant (TT+TC/CC) & 0: TT+TC; 7: TC/CC & 3: TT+TC; 39: TC/CC & 1.0000 \\
\hline & & & Recessive (TT/TC+CC) & 0: TT/TC; 7: TC+CC & 1: TT/TC; 41: TC+CC & 1.0000 \\
\hline rs9096 & $\mathrm{T}$ & $\mathrm{C}$ & Genotype (CC/CT/TT) & 0: CC; 1: CT; 6: TT & 1: CC; 6: CT; 35: TT & 1.0000 \\
\hline & & & Allele (C/T) & 1: C; 13: T & 8: C; 76: T & 1.0000 \\
\hline & & & Dominant (CC+CT/TT) & 1: CC+CT; 6: CT/TT & 7: CC+CT; 35: CT/TT & 1.0000 \\
\hline & & & Recessive (CC/CT+TT) & 0: CC/CT; 7: CT+TT & 1: CC/CT; 41: CT+TT & 1.0000 \\
\hline
\end{tabular}

\section{DISCUSSION}

In our previous study, not all of the WD cases were confirmed molecularly [15]. One explanation of undiagnosed WD in a molecular level could be the fact that possibly WD patients with clinically confirmed WD diagnosis actually might have Wilson's disease-like disease. Wilson's disease clinical and laboratory diagnostic criteria were developed in 2001 and have not changed since. Several studies have been published on the revision of the guidelines and criteria [20] but remain unchanged at the European level [11]. Two of the criteria for WD diagnostics are reduced levels of ceruloplasmin in the blood and neurological symptoms. In case of WD, the levels of ceru-loplasmin are reduced due to copper accumulation, but other causes, such as aceruloplasminemia and malabsorption, may also cause reduced levels of ceruloplasmin [11]. Detection of ceruloplasmin level in the blood is recommended as a first step in WD diagnostics [21]. Very low levels of ceruloplasmin in the blood $(<5 \mathrm{mg} / \mathrm{dL})$ are highly associated with WD, but such low levels may also be found in other
[8] and AFib $[9,10]$, concluding that changes in the $C P$ gene promoter may be associated with altered levels of ceruloplasmin. Analyzing the $C P$ gene promoter in the current study, it was concluded, that the AA genotype of the rs66508328 variant and the GG genotype of rs 11708215 (both according to the recessive inheritance model) were more common in patients who were not confirmed molecularly to have WD according to the $A T P 7 B$ genotype. This may indicate that the above-mentioned variants in the $C P$ gene could affect gene expression, resulting in reduced levels of ceruloplasmin in the blood, that in turn, leads to increased accumulation of iron in the brain, causing Parkinson's disease-like symptoms, which are also characteristic of WD. The results observed here points to a further development of the study by increasing the size of the patient group, and although the differences were statistically significant, due to the small size of the groups, the data could have been accidental.

Considering the important interaction of ATOX1 protein with ATP7B protein, there have been several studies looking for possible changes in the ATOX1 gene in WD 
patients, but so far, the results have been negative [ 5,1$]$. In this study, there were no identified changes in the ATOXI gene causing functional changes of the protein, so we were unable to prove that changes in the ATOXI gene could modify the WD phenotype.

In studies of changes in other genes that could affect the clinical manifestations of WD, the researchers focused on the COMMD1 gene, in which pathogenic variants have been shown to cause copper toxicosis in Bedlington terriers that is clinically similar to WD in humans [23]. There have been several studies analyzing the possible effects of this gene variants on humans. Stuehler et al. [24] revealed that the substitution of the nucleotide in the position c.492T $>C$ (p.Asn164 or rs9096) is associated with earlier (about 10 years) expression of clinical symptoms in WD patients. In this study, the above-described variant was also found in Latvian patients. Analyzing its possible association with clinical manifestations of the disease, the relationship was not found, so the above observation was not confirmed. In another study in 2010, Gupta et al. [6] reported the effect of another variant, c.521C $>$ T (p.Thr 174Met; rs139775239), on the WD phenotype; they associate this variant with more elevated levels of copper in the urine, as well as with enhanced cell apoptosis in WD patients. In the current study, no such variant was found in the COMMD1 gene in any of the Latvian patients. There have been several studies with the effect of COMMD1 gene variants on WD, but unfortunately, no association with these studies was confirmed $[25,26]$, the same as in present study.

\section{CONCLUSIONS}

1) The difference variants of the $C P$ gene contribute to a WD-like phenotype in clinically confirmed WD patients with neurological symptoms and without pathogenic variants in the $A T P 7 B$ gene, but for confirmation, a larger study group is required; 2) no changes were found in the ATOX1 gene that would cause functional changes of the protein; 3 ) no statistically significant differences were found between allele and genotype frequencies in the COMMD1 gene and the first clinical manifestations of WD; 4) allelic variants in the ATOX1 and COMMD1 genes do not modify the clinical manifestation of WD in Latvian patients.

Declaration of Interest. The authors report no conflicts of interest. The authors alone are responsible for the content and writing of this article.

Funding. This study was partially financed by a grant of Riga Stradiņš University, Department of Doctoral studies and grant of Roche Academy.

\section{REFERENCES}

1. Członkowska A, Litwin T, Dusek P, Ferenci P, Lutsenko S, Medici V, et al. Wilson disease. Nat Rev Dis Prim. 2018; 4(1): 21.

2. Ala A, Schilsky M. Genetic modifiers of liver injury in hereditary liver disease. Semin Liver Dis. 2011; 31(2): 208-214.

3. Medici V, Weiss K-H. Genetic and environmental modifiers of Wilson disease. In: Członkowska A, Schilsky ML, Editors. Handbook of Clinical Neurology. Amsterdam, The Netherlands: Elsevier. 2017; 142: 35-41.

4. Kluska A, Kulecka M, Litwin T, Dziezyc K, Balabas A, Piatkowska M, et al. Whole-exome sequencing identifies novel pathogenic variants across the $A T P 7 B$ gene and some modifiers of Wilson's disease phenotype. Liver Int. 2019; 39(1): 177-186.

5. Simon I, Schaefer M, Reichert J, Stremmel W. Analysis of the human Atox 1 homologue in Wilson patients. World J Gastroenterol. 2008; 14(15): 2383-2387.

6. Gupta A, Chattopadhyay I, Mukherjee S, Sengupta M, Das SK, Ray K. A novel COMMD1 mutation Thr174 Met associated with elevated urinary copper and signs of enhanced apoptotic cell death in a Wilson Disease patient. Behav Brain Funct. 2010; 6: 33.

7. Patel BN, Dunn RJ, Jeong SY, Zhu Q, Julien J-P, David S. Ceruloplasmin regulates iron levels in the CNS and prevents free radical injury. J Neurosci. 2002; 22(15): 6578-6586.

8. Zhao N, Xiao J, Zheng Z, Fei G, Zhang F, Jin L, et $a l$. Single-nucleotide polymorphisms and haplotypes of non-coding area in the $\mathrm{CP}$ gene are correlated with Parkinson's disease. Neurosci Bull. 2015; 31(2); 245 256.

9. Adamsson Eryd S, Sjögren M, Smith JG, Nilsson PM, Melander O, Hedblad B, et al. Ceruloplasmin and atrial fibrillation: Evidence of causality from a population-based Mendelian randomization study. J Intern Med. 2014; 275(2): 164-171.

10. Arenas de Larriva AP, Norby FL, Chen LY, Soliman EZ, Hoogeveen RC, Arking DE, et al. Circulating ceruloplasmin, ceruloplasmin-associated genes, and the incidence of atrial fibrillation in the atherosclerosis risk in communities study. Int J Cardiol. 2017; 241: 223-228.

11. Ferenci P, Czlonkowska A, Stremmel W, Houwen R, Rosenberg W, Schilsky M, et al.; EASL Clinical Practice Guidelines: Wilson's disease. J Hepatol. 2012; 56(3): 671-685. 2012. 
12. Gromadzka G, Schmidt HHJ, Genschel J, Bochow B, Rodo M, Tamacka B, et al. Frameshift and nonsense mutations in the gene for ATPase7B are associated with severe impairment of copper metabolism and with an early clinical manifestation of Wilson's disease. Clin Genet. 2005; 68(6): 524-532.

13. Poláková H, Katrincsáková B, Minárik G, Ferakova, E, Ficek A, Baldovic M, et al. Detection of His1069Gln mutation in Wilson disease by bidirectional PCR amplification of specific alleles (BI-PASA) test. Gen Physiol Biophys. 2007; 26(2): 91-96.

14. Krumina A, Keiss J, Sondore V, Chernushenko A, Cernevska G, Zarina A, et al. From clinical and biochemical to molecular genetic diagnosis of Wilson disease in Latvia. Genetika. 2008; 44(10): 1379-1384.

15. Zarina A, Tolmane I, Kreile M, Chernushenko A, Cernevska G, Pukite I, et al. Genetic variation spectrum in ATP7B gene identified in Latvian patients with Wilson disease. Mol Genet Genomic Med. 2017; 5(4): 405-409.

16. gnomAD. [Online]. Available: https://gnomad. broadinstitute.org/. [Accessed: April 15 2019].

17. Chr13: 52.51M-52.51M, 1000 Genomes Browser. [Online]. Available: https://www.ncbi.nlm.nih.gov/ varia tion/tools/1000genomes/?gts $=$ rs 1057516740 . [Accessed: October 16 2018].

18. Richards S, Aziz N, Bale S, Bick D, Das S, GastierFoster J, et al. Standards and guidelines for the interpretation of sequence variants: A joint consensus recommendation of the American College of Medical Genetics and Genomics and the Association for Molecular Pathology. Genet Med. 2015; 17(5): 405-424.
19. Human Splicing Finder, Version 3.1. [Online]. Available: http://www.umd.be/HSF3/. [Accessed: October 18 2018].

20. Hedera P. Update on the clinical management of Wilson's disease. Appl Clin Genet. 2017; 10: 9-19.

21. Roberts EA, Schilsky ML. Diagnosis and treatment of Wilson disease: An update. Hepatology. 2008; 47(6): 2089-2111.

22. Hellman NE, Gitlin JD. Ceruloplasmin metabolism and function. Annu Rev Nutr. 2002; 22(1): 439-458.

23. van De Sluis B, Rothuizen J, Pearson PL, van Oost $\mathrm{BA}$, Wijmenga C. Identification of a new copper metabolism gene by positional cloning in a purebred dog population. Hum Mol Genet. 2002; 11(2): 165-173.

24. Stuehler B, Reichert J, Stremmel W, Schaefer M. Analysis of the human homologue of the canine copper toxicosis gene MURR1 in Wilson disease patients. J Mol Med. 2004; 82(9): 629-634.

25. Lovicu M, Dessi V, Lepori MB, Zappu A, Zancan L, Giacchino R, et al.. The canine copper toxicosis gene MURR1 is not implicated in the pathogenesis of Wilson disease. J Gastroenterol. 2006; 41(6): 582587.

26. Weiss KH, Merle U, Schaefer M, Ferenci P, Fullekrug J, Stremmel W. Copper toxicosis gene MURR1 is not changed in Wilson disease patients with normal blood ceruloplasmin levels. World J Gastroenterol. 2006; 12(14): 2239-2242. 